\title{
Features of energetic particle radial profiles inferred from geosynchronous responses to solar wind dynamic pressure enhancements
}

\author{
Y. Shi, E. Zesta, and L. R. Lyons \\ Department of Atmospheric and Oceanic Sciences, University of California, Los Angeles, 405 Hilgard Ave, Los Angeles, CA \\ 90095-1565, USA
}

Received: 7 September 2008 - Revised: 14 January 2009 - Accepted: 27 January 2009 - Published: 19 February 2009

\begin{abstract}
Determination of the radial profile of phase space density of relativistic electrons at constant adiabatic invariants is crucial for identifying the source for them within the outer radiation belt. The commonly used method is to convert flux observed at fixed energy to phase space density at constant first, second and third adiabatic invariants, which requires an empirical global magnetic field model and thus might produce some uncertainties in the final results. From a different perspective, in this paper we indirectly infer the shape of the radial profile of phase space density of relativistic electrons near the geosynchronous region by statistically examining the geosynchronous energetic flux response to 128 solar wind dynamic pressure enhancements during the years 2000 to 2003 . We thus avoid the disadvantage of using empirical magnetic field models. Our results show that the flux response is species and energy dependent. For protons and low-energy electrons, the primary response to magnetospheric compression is an increase in flux at geosynchronous orbit. For relativistic electrons, the dominant response is a decrease in flux, which implies that the phase space density decreases toward increasing radial distance at geosynchronous orbit and leads to a local peak inside of geosynchronous orbit. The flux response of protons and nonrelativistic electrons could result from a phase density that increases toward increasing radial distance, but this cannot be determined for sure due to the particle energization associated with pressure enhancements. Our results for relativistic electrons are consistent with previous results obtained using magnetic field models, thus providing additional confirmation that these results are correct and indicating that they are not the result of errors in their selected magnetic field model.
\end{abstract}

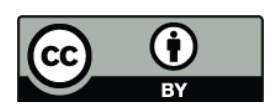

Correspondence to: Y. Shi

(yongshi@atmos.ucla.edu)
Keywords. Magnetospheric physics (Energetic particles, trapped; Magnetospheric configuration and dynamics; Solar wind-magnetosphere interactions)

\section{Introduction}

Enhancements of relativistic electron fluxes in the outer radiation belt have been frequently observed during geomagnetic disturbances (Friedel et al., 2002, and references therein). The processes that accelerate these electrons have received much attention by researchers. Recent proposals fall into two categories. One attributes the acceleration to the transport of a population of electrons from the outer magnetosphere by radial diffusion, which has been referred to as an external acceleration mechanism, and the other relies on the acceleration of the electron population already present in the inner magnetosphere through wave-particle interactions, which was referred to as an internal acceleration mechanism (Freidel et al., 2002; Green and Kivelson, 2004; Chen et al., 2005, 2007, and reference therein; Summers et al., 2007; Hudson et al., 2008). Different mechanisms produce different radial gradients of phase space density as a function of adiabatic invariants. Radial diffusion causes a radial profile with positive gradient in the vicinity of geosynchronous, while internal acceleration generally leads to a peak inside of geosynchronous. Deriving the radial profile of phase space density in terms of three adiabatic invariants and tracing its time development is crucial in identifying different accelerations of relativistic electrons in the outer radiation belt. The commonly used method is to convert the flux observed at fixed energy to phase space density at constant first, second and third adiabatic invariants. Many papers have been written on this topic recently (Selesnick and Blake, 1997, 2000; Hilmer et al., 2000; McAdams et al., 2001; Green and Kivelson,

Published by Copernicus Publications on behalf of the European Geosciences Union. 
2004; Onsager et al., 2004; Taylor et al., 2004; Iles et al., 2006; Chen et al., 2005, 2007).

One of the possible uncertainties that might be introduced in those studies is associated with the global magnetic field model chosen to calculate the adiabatic invariants when deriving phase space density as a function of them (Green and Kivelson, 2004; Chen et al., 2005, 2007). For example, Green and Kivelson (2004) derived the radial profile of phase space density of relativistic electrons at constant first and second adiabatic invariants using data from the POLAR spacecraft along with the Tsyganenko geomagnetic field model (T96) (Tsyganenko and Stern, 1996) for the time intervals before and during magnetic storms. They found that the dayside radial profile has a peak inside geosynchronous orbit, which is consistent with an internal acceleration mechanism, but that the night side radial profile has a phase space density maximum always outside of geosynchronous, which is more consistent with an external acceleration mechanism. Green and Kivelson (2004) argued that the inconsistency between dayside and night side comes from the imperfection of the magnetic field model used in their study, and they concluded that relativistic electrons are accelerated primarily by an internal acceleration mechanism earthward of geosynchronous orbit during magnetic storms. Chen et al. (2005) calculated phase space density profiles in the geosynchronous region and used a variety of magnetic field models to show how results could be substantially different based on the magnetic field model. It is clear that such results for phase space density radial profiles are strongly magnetic field model dependent.

Chen et al. (2005) determined the relativistic electron phase space density at geosynchronous by using multisatellite observations of energetic electron fluxes from geosynchronous satellites during quiet times. The reason they chose quiet times was to minimize the influence of the empirical magnetic field models used in their study due to the relative lack of dynamic variation of the Earth's geomagnetic field during quiet times and the models being more capable of reproducing quiet-time magnetic fields than disturbed magnetic fields. They found that the quiet-time radial profile of electron phase space density is energy-dependent. Lowenergy electrons have a positive or flat slope in the vicinity of geosynchronous, while high-energy electrons have a negative slope beginning from some location within geosynchronous orbit. Similar to Green and Kivelson (2004), their results were also sensitive to the magnetic field model choice and the error causing by the two different magnetic field models used in their paper was estimated.

More recently, Chen et al. (2007) found that the radial profiles of relativistic electrons under storm main phase, recovery phase, non-storm phase (or quiet times) and overall conditions all have a peak inside of geosynchronous orbit. More importantly, Chen et al. (2007) monitored the radial PSD profiles in time and found that the peaks earthward of geosynchronous are persistent in time, indicating that an external source of acceleration (radial diffusion) is unlikely, as that could provide the occasional negative slopes at geosynchronous but the positive slopes should dominate in time. Therefore, Chen et al. (2007) argued that such frequent and persistent peaks in equatorial electron PSD near or inside geosynchronous orbit provide unambiguous evidence for local wave-particle acceleration. Still, the results of this study are dependent on empirical magnetic field models, even though the authors did take extra care to minimize that uncertainty. Thus, more evidence is needed, especially evidence that is not magnetic-field-dependent. At the same time, further evidence on the gradient of radial profiles under quiet time conditions is also worthy of investigation. In this paper, we address these two issues from a different perspective than the previous works by examining the geosynchronous flux response to solar wind dynamic pressure enhancements.

Solar wind dynamic pressure enhancements can increase the magnetospheric magnetic field, which is associated with an induced electric field that moves particles earthward and energizes the particles (Shi et al., 2005, 2006). This leads to significant disturbances of the energetic particle fluxes in the inner magnetosphere ( $\mathrm{Li}$ et al., 1993, 1998, 2003; Hudson et al., 1998; Lee and Lyons, 2005). Figure 1 shows an example of a typical response of the geosynchronous electron flux to an abrupt solar wind pressure enhancement occurring on 18 August 2002. The solar wind data is from ACE spacecraft and propagated to $17 R_{E}$ using the Weimer's solar wind propagation technique (Weimer et al., 2003). The vertical lines indicate the onset of the pressure enhancement. We plot nine energy channels ranging from $50-75 \mathrm{keV}$ to 1.1 to $1.5 \mathrm{MeV}$ from the top to bottom in each panel. Notice that the low-energy channels show a flux increase, whereas the high-energy channels show a flux decrease. For protons (figure not shown here), all energy channels show a flux increase. This pressure enhancement occurred during a nonstorm time (see the SYM-H panel in Fig. 1). It is clear that the responses are species and energy dependent. Since solar wind dynamic pressure enhancements compress the magnetosphere, they transport particles inward from larger radial distance while conserving the first $(\mu)$ and second $(J)$ adiabatic invariants. The combination of the pre-existing radial distribution of phase space density at constant $\mu$ and $J$ and the adiabatic acceleration of particles thus determines the flux response observed at geosynchronous orbit. Therefore the response of the energetic particle fluxes at geosynchronous reveals information on the radial profile of their phase space density just prior to compression.

Since different acceleration mechanisms are expected to lead to different radial profiles of phase space density, the geosynchronous flux responses can thus be used as an indicator of the different mechanisms. However, for a conclusive determination of the acceleration mechanism, observation of the time development of the radial PSD profiles is necessary. This is not possible with the technique presented in this paper. 


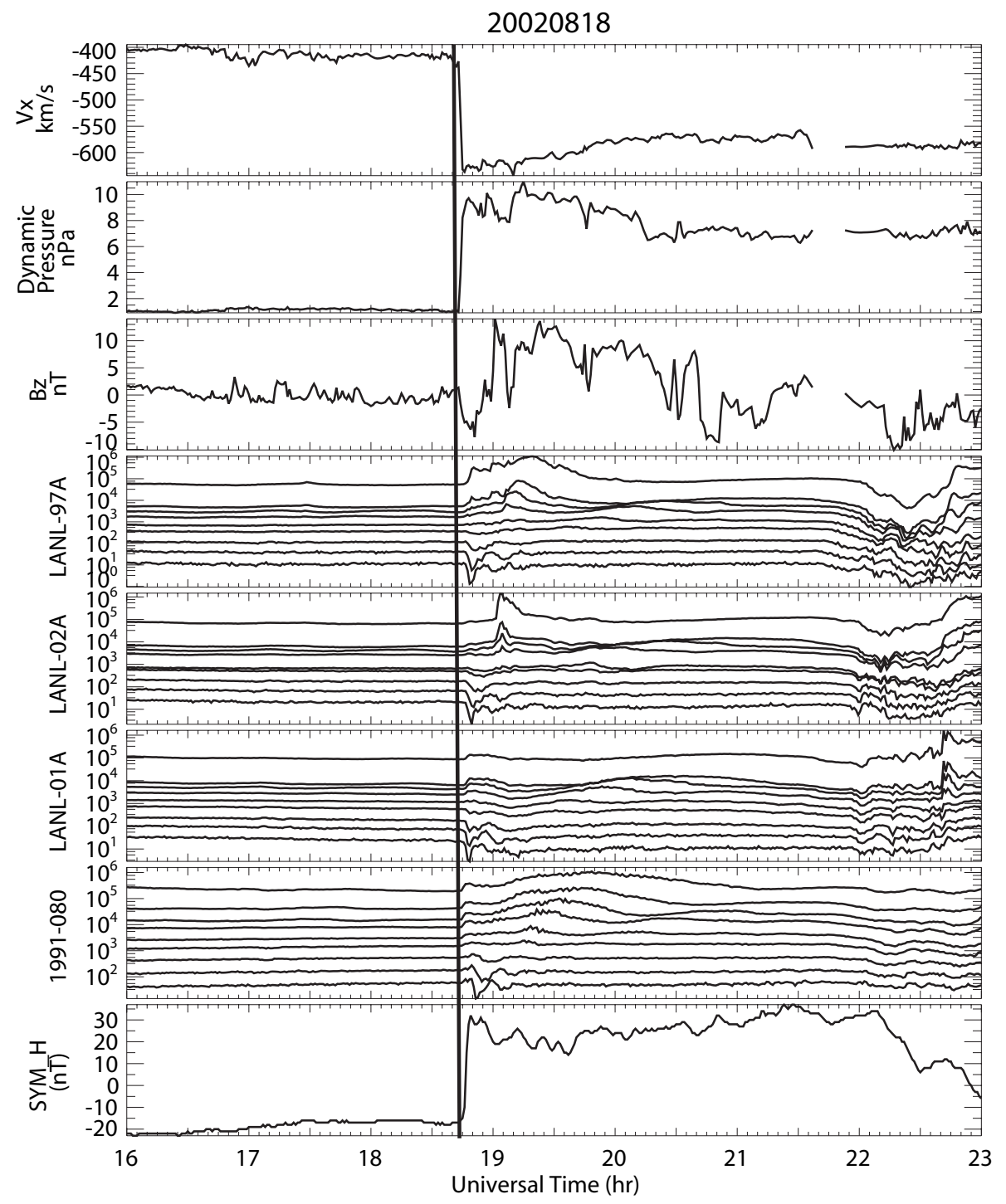

Fig. 1. An example of typical response of geosynchronous electron flux to a solar wind pressure enhancement occurring on 18 August 2002. The solar wind data is from ACE spacecraft and propagated to $17 R_{E}$. The fluxes of 9 energy channels ranging from 50-75 keV to 1.1 to $1.5 \mathrm{MeV}$ from the top to the bottom of each panel are plotted for each available LANL satellite.

When evaluating the response of geosynchronous fluxes to magnetospheric compressions it is important to note that a detector measuring flux at a constant energy at a fixed location may see somewhat lower $\mu$ particles after a pressure increase than before because of the increase in the magnetic field. It is thus necessary to take into consideration the fact the phase space density decreases with increasing $\mu$ when inferring information on the particle radial profiles. We will explore this further in the Discussion section.

Although the method we employ in this paper cannot quantitatively derive phase space density gradients, as did the previous papers by using a magnetic field model, it can provide indirect evidence of some of the features of radial profile from a different perspective and gives results that are not magnetic field model dependent.
We statistically investigate the response of geosynchronous energetic particle fluxes to 128 abrupt solar wind dynamic pressure enhancements. It has been know for many years that there is an equilibrium structure of radiation belt electrons during quiet times that represent a balance between pitch-angle scattering losses and radial diffusion (Lyons and Thorne, 1973). This implies that radial diffusion is essentially continuous. Furthermore, local acceleration is believed to result from electrons convecting into the near-Earth plasma sheet in association with convection. This is also a process that happens essentially continuously. It is only a matter of the intensity of the process, relative to losses, that gives the well-known enhancements of relativistic electrons. During more geomagnetically active times the processes of convection and radial diffusion are naturally even 

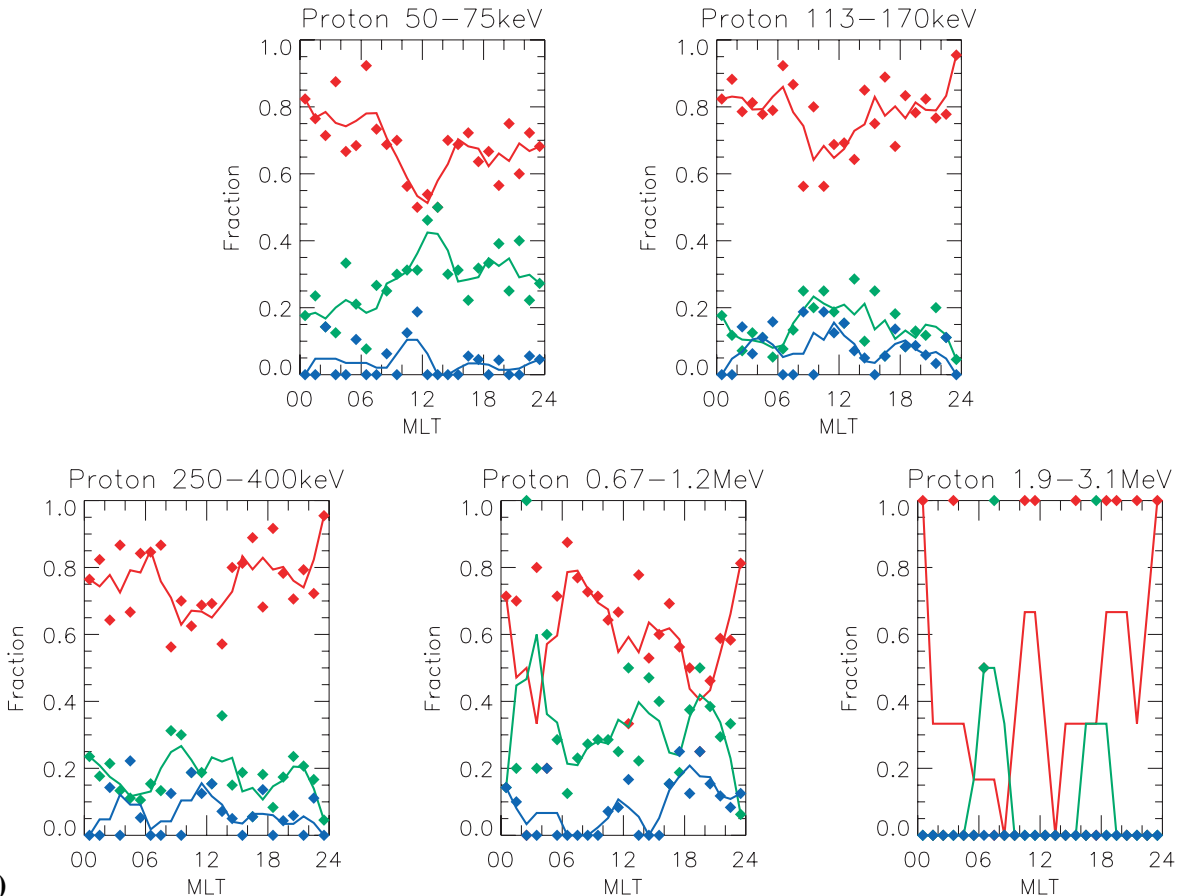

(a)
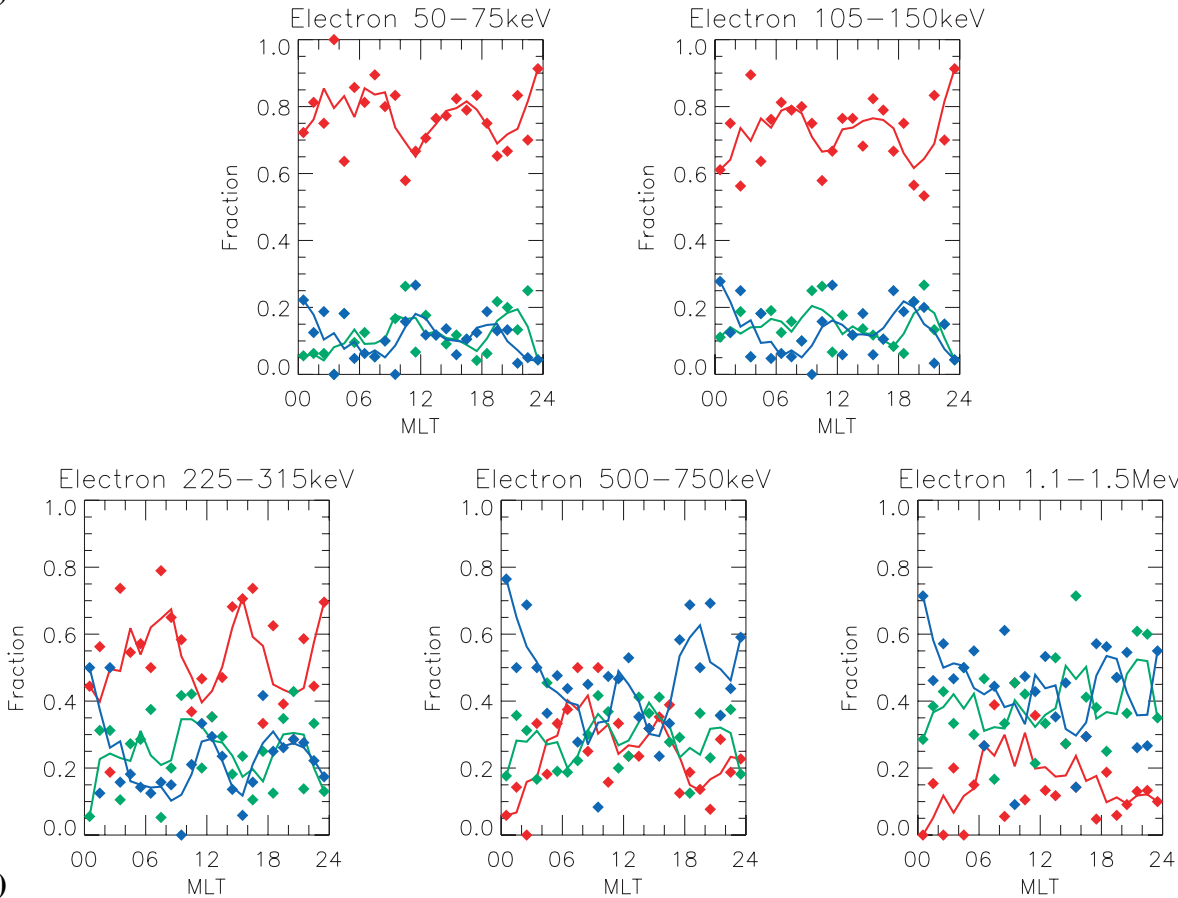

Fig. 2. (a) Local-time distribution of proton flux responses to the dynamic pressure enhancements for 5 selected individual energy channels. The red lines represent percentage of events showing flux increase; the green ones are percentage of events showing no discernible flux change and the blue ones are percentage of events showing flux decrease. (b) The same as panel (a), except that it if for electrons.

more enhanced. A compression event due to solar wind dynamic pressure enhancements reveals the PSD profile that existed before the compression. Since radial diffusion and convection occur continuously, a significant statistical sample of compression events will statistically reveal the PSD profiles under a variety of geomagnetic conditions. We present the statistical results for all of our events occurring during both storm times (i.e. main and recovery phase) and non-storm times, and the results only for non-storm time events. We use the response to infer information on the radial profile 
of phase space density for different energy channels and species, and discuss the implications of the inferred radial profile on the acceleration of relativistic electrons for nonstorm times as well as storm times and compare this to what is found for lower energy electrons and protons

\section{Data}

We use the propagated solar wind data (Weimer et al., 2003) to identify the pressure enhancements and the relevant solar wind and IMF conditions. We select all available pressure enhancements during the years of 2001 to 2003 that satisfy the following criteria.

- The change of the dynamic pressure is sharp, i.e. sudden and rapid pressure jump. The duration of period of high pressure is at least $30 \mathrm{~min}$.

- The increase in pressure is $\Delta P \geq 1 \mathrm{nPa}$ and $\Delta P / P_{0} \geq 0.5$, where $P_{0}$ is the pressure prior to the enhancement.

- Observations are available from at least two LANL spacecrafts.

The application of the above criteria resulted in 128 pressure enhancement events.

We use the LANL energetic particle fluxes obtained from geosynchronous satellites. We use the SYM-H index (Iyemori and Rao, 1996) to identify magnetic storms occurring during the above period. Our statistics show that $62 \%$ of the pressure enhancements occurred during quiet times, $22 \%$ at storm sudden commencement and $16 \%$ during a storm main phase or recovery phase. In this study, magnetic storms are chosen as those with minimum SYM-H less than $-50 \mathrm{nT}$. For each one of the 128 pressure enhancement events, we recorde the flux response (increase, decrease, or no discernible change) of each energy channel for both electrons and protons and with respect to the MLT location of the observing LANL spacecraft that measured that particular flux.

\section{Statistical results and discussion}

Figure 2a shows the local-time distribution of the responses of proton fluxes to solar wind dynamic pressure enhancements for 5 selected individual energy channels for all 128 events. The red dots give the percentage of flux increase events, the green dots give the percentage of no discernible flux change events, and the blue dots give the percentage of flux decrease events. To more clearly show the trends, lines are drawn that give the three-point boxcar average of successive points for each panel. It is clear that the dominant response for each energy channel for protons is a flux increase, the number of flux increase events significantly exceeding the number of flux decrease events. For the 1.9-3.1 MeV channel, most of the measurements were below the background so the event sample is not large enough for the results to have statistical significance.

Figure $2 \mathrm{~b}$ shows the local-time distribution of electron flux responses for 5 selected individual energy channels. It is clear that for low energy electrons, the dominant response is a flux increase. However, for relativistic electrons ( $>500 \mathrm{keV})$, the primary response is a flux decrease, the number of flux decrease events substantially exceeding the number of flux increase events. We also see a noon-midnight asymmetry for the flux decrease events, more flux decrease events occurring on night side than on day side.

GOES 10 observations of $>0.6 \mathrm{MeV}$ electrons and $0.7-$ $4 \mathrm{MeV}$ protons (figures not shown here) during the same pressure enhancements give the same statistical results, namely the dominance of proton flux increases at all energies, and of relativistic electron flux decreases. Note that we have only evaluated the direct compression effect of pressure enhancements on the particle flux by comparing the flux just before and after the pressure enhancements. Later flux variations after the initial compression, such as considered by $\mathrm{Li}$ et al. (2003), are not considered here.

We consider for simplicity only equatorially mirroring particles and assume that $\mu$ is conserved in the following discussion. This is a reasonable assumption for the energetic electrons at geosynchronous, since energetic electrons generally undergo weak pitch angle diffusion except very near the outer boundary of trapping (Lyons, 1997, and references therein). The assumption is less realistic for protons, since current sheet scattering can bring them closer to isotropy (Lyons, 1997). However, the arguments below also hold for isotropic distributions, except that they apply at constant energy invariant $\lambda=W_{k} V^{2 / 3}$ (Wolf, 1983; Wang et al., 2001) instead of at constant $\mu$, where $W_{k}$ is particle energy and $V$ is flux tube volume.

Considering only the radial displacement effect first, for protons and low-energy electrons, the statistics imply that the phase space density at the onset of the pressure enhancements at geosynchronous orbit often increased with increasing radial distance $L$. Figure 3 a schematically shows the inferred radial profile of phase space density at constant $\mu$ and $J$ (presumed in this discussion to be 0 ) prior to and after the onset of the pressure enhancements suggested by our statistics. The black line shows the profile prior to the onset of pressure enhancements, which is assumed to have a peak somewhere beyond geosynchronous orbit. The grey line is the profile after the onset when the distribution has been pushed earthward with the compression, which shows how the LANL instruments would observe the population with larger phase space density that was originally outside of geosynchronous orbit. This shows how such a radial profile would lead to an enhancement of particle flux at geosynchronous orbit. 


\section{Proton and low-energy electron}

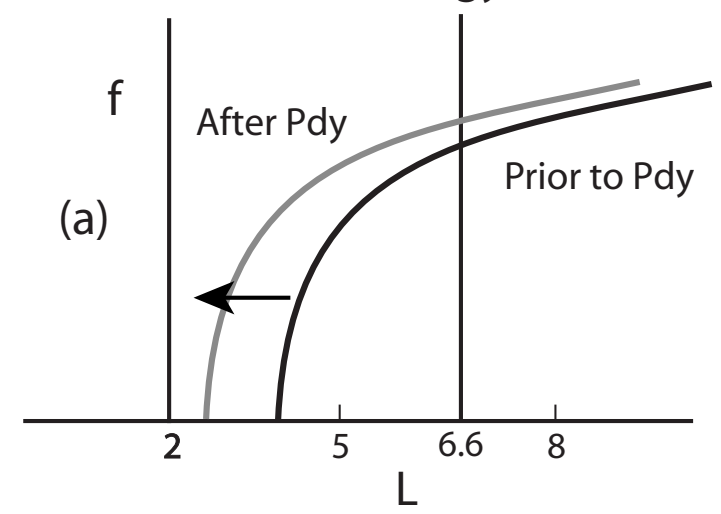

Relativistic electron

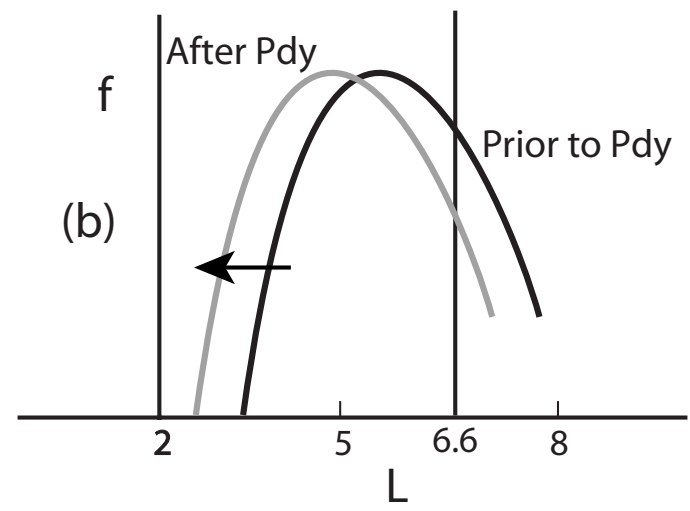

Fig. 3. Schematic radial profiles of phase space density at constant $\mu$ and $J$ inferred from the particles' response to the dynamic pressure enhancements. (a) For protons and low-energy electrons; (b) For relativistic electrons. The black lines represent the profile prior to the pressure enhancements. The grey lines represent the profile after the onset of the pressure enhancements.

Now, we consider that compression can increase the magnetic field, which can dramatically alter the above simplified inference. Since the particle detectors measure fluxes within fixed energy channels, the $\mu$ values measured by the detectors may actually be somewhat reduced after the pressure enhancement, which by itself would be expected to lead to a flux increase because of the increase in energetic particle fluxes with decreasing energy. Thus we cannot be sure that the radial profiles at fixed $\mu$ actually increased with increasing radial distance for each case where an increase in fluxes was observed. For example, even if the radial profile were flat before compression, the adiabatic acceleration would still lead to small flux enhancements. On the other hand, if the radial profile actually had a negative slope beyond geosynchronous, we could still observe a flux enhancement if the flux enhancement from the decrease in $\mu$ values exceeded the flux decrease due to the radial displacement. At this point, we cannot test the extent to which this occurred only based on our data and we are therefore not able to draw any conclusions on the PSD radial profiles of protons or non-relativistic electrons. Despite this limitation, the contrast with the relativistic electrons discussed below remains.

For relativistic electrons, the statistics imply that the phase space density at the onset of the pressure enhancements at geosynchronous obit often decreased with increasing radial distance $L$. Figure $3 \mathrm{~b}$ schematically shows the radial profile of relativistic electrons. It illustrates how, after the onset of a pressure enhancement, the LANL instruments would observe the population with smaller phase space density originally outside of geosynchronous orbit, resulting in a decrease of relativistic electron fluxes at geosynchronous orbit, based solely on radial adiabatic displacement. Since the detectors actually measured a somewhat smaller $\mu$ after the compressions, which by itself would lead to flux enhancements, we can be confident of the inferred phase space density decrease with increasing radial distance and the contrast with the radial profiles of protons and lower energy electrons. This indicates that the radial profile of relativistic electrons often peaks earthward of geosynchronous orbit. While we are not able to conclusively differentiate between an internal or external acceleration mechanism (we do not monitor the time development of the PSD profile), our result that relativistic electrons often peaks inside of the geosynchronous orbit is consistent with the results of Green and Kivelson (2004) and suggests that the result often applies to non-storm conditions, which, in turn, is consistent with the results of Chen et al. $(2005,2007)$. Our results thus complement these earlier results in showing that the relativistic electron PSD statistically has a peak earthward of geosynchronous and it is not magnetic field model dependent.

In addition, Lyatsky and Khazanov (2008) found that the short time scale flux variation of relativistic electrons at geosynchronous orbit has negative correlation with increasing solar wind density. Since number density is the major contributor to dynamic pressure, our results that solar wind dynamic pressure enhancements cause decreasing relativistic electron flux at geosynchronous are consistent with Lyatsky and Khazanov (2008).

To demonstrate that the results inferred from Fig. 2 are not prejudiced by the inclusion of stormtime events, Fig. 4 shows a comparison of protons and electron statistics for two selected channels for the non-stormtime events, where we have excluded events occurring during the main and recovery phases of the identified magnetic storms. Storm sudden commencement events are included, because they reflect the pre-storm radial profiles. It is clear that, for protons and lowenergy electrons, the dominant response to a compression is also a flux increase. For relativistic electrons, however, the primary response is a flux decrease. The statistics for non-storm conditions in Fig. 4 is quite similar to the overall statistics shown in Fig. 2a and b, implying that the inferences above concerning the radial profiles of phase space density indeed do apply to non-storm conditions. 

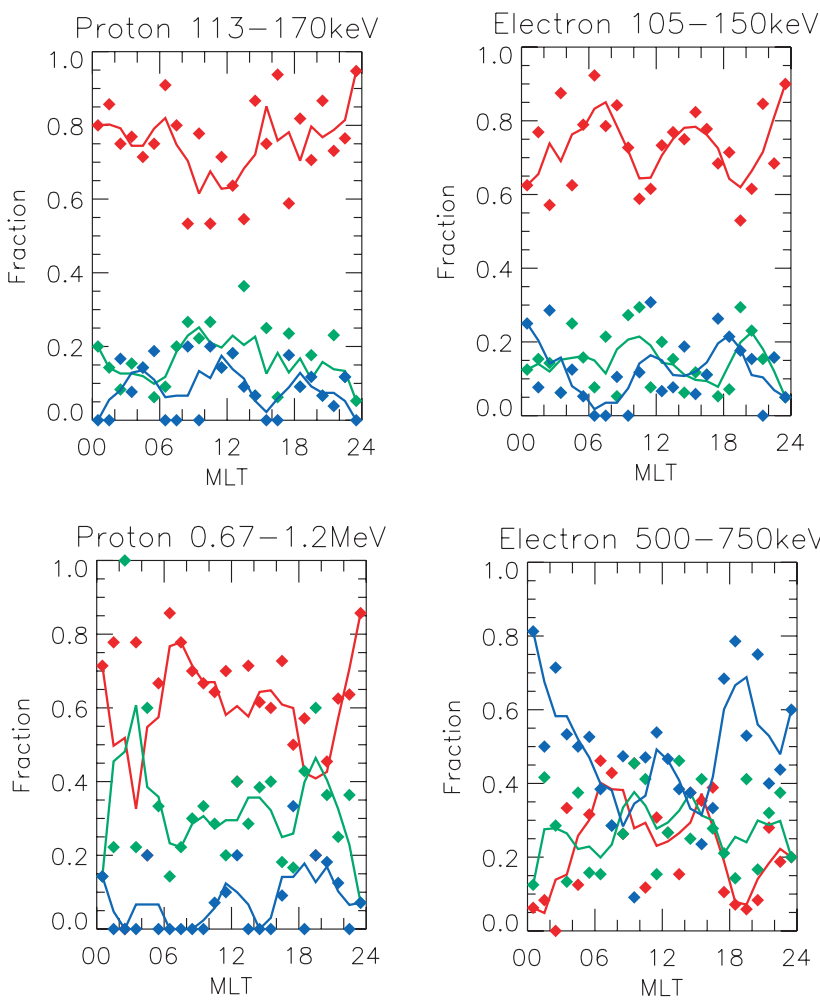

Fig. 4. Local-time distribution of proton and electron flux response to the dynamic pressure enhancements for 2 selected channels each for protons and electrons during non-storm times.

Note that we do not have enough storm-time events (only 20 cases) to show the detailed local time distribution of each flux variation category as shown in Figs. 2 and 4. But, the storm-time plots do show the same trend of each flux variation as the non-storm and the overall cases shown in Figs. 4 and 2, i.e. a flux decrease is dominant for relativistic electrons, but flux increases dominate for low energy electrons and for protons. Figure 5 shows the statistics for the storm time events. The percentage of flux increase, decrease and no change events averaged over all MLT is shown instead of the fitting curves as functions of MLT, due to lack of sufficient samples. It is clear that the trend for each flux variation category of electrons and protons is the same as for the non-storm and overall statistics shown in Figs. 4 and 2. These results thus suggest that, statistically, consistent with the results of Chen et al. (2007), the peak of the relativistic electron PSD is inward of geosynchronous. While Chen et al. (2007) were able to reliably attribute the peak of PSD to an internal acceleration mechanism, we are not able to make such a determination here. The fact that the majority of relativistic electron fluxes decrease after a compression, does suggest an internal acceleration mechanism for at least part of the time. However, Figs. 2 and 4 do indicate a sizeable number of events with relativistic electron flux increases, so sudden changes in radial diffusion cannot be discounted as
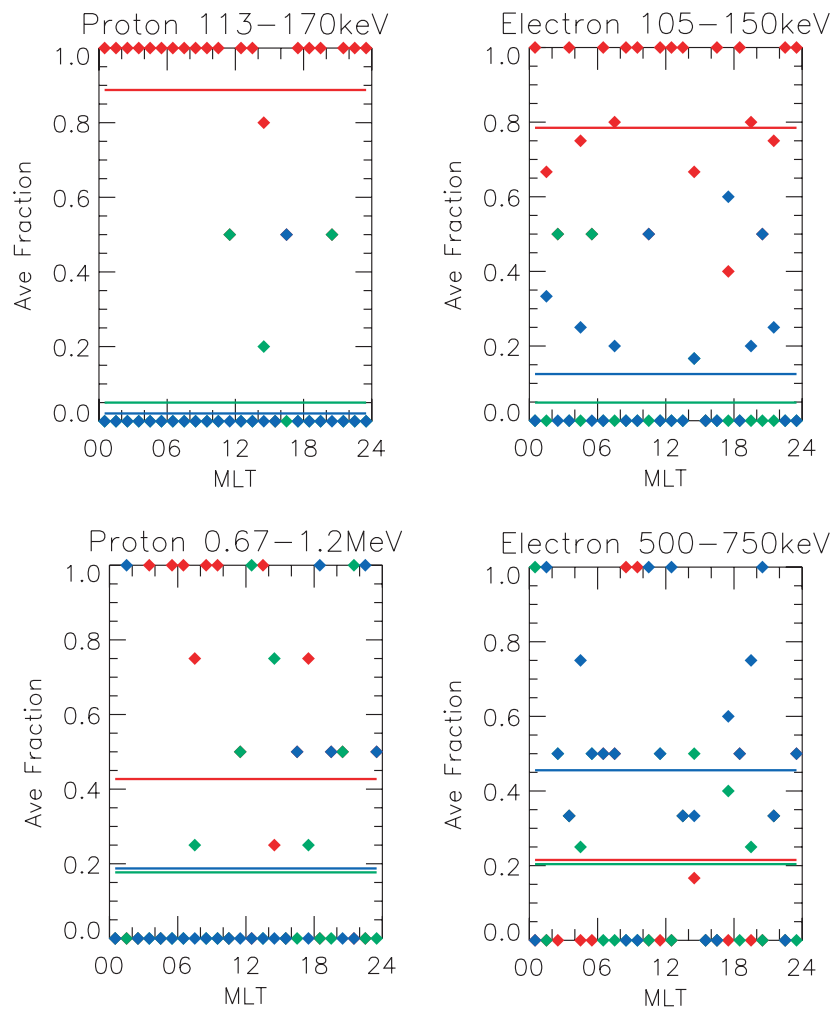

Fig. 5. The same as Fig. 4, except that it is for storm times and that it plots the averaged percentage of each flux variation category instead of the fitting curves.

the source of the PSD peak inward of geosynchronous, for possibly a smaller part of the time.

There are several wave-particle interaction models proposed to be the possible internal acceleration mechanisms (Friedel et al., 2002). However, our analysis does not give new information that would help distinguish between the different proposals.

Our statistics also show a significant noon-midnight asymmetry of flux decrease events for relativistic electrons (see bottom middle panel of Fig. $2 b$, and bottom right panel of Fig. 4), i.e. more flux decrease events occurring on the night side than on the dayside. This is consistent with the asymmetry of magnetic drift paths of equatorially mirroring electrons, which drift following constant magnetic field contours. The magnetic field is typically more stretched near midnight and more compressed near noon. Furthermore, studies on the response of the magnetic field at geosynchronous orbit to solar wind dynamic pressure changes (Wind and Sibeck, 1997; Borodkova et al., 2005; Wang et al., 2008) suggest that the magnetic field increases more near noon than on the night side as a response to solar wind dynamic pressure enhancements, which will add to the day-night magnetic field asymmetry. This asymmetry causes electrons of a particular energy to move outward at noon and inward at midnight relative to their drift trajectories in a dipole magnetic field. This 
suggests that, statistically, the peak of radial profile of relativistic electrons is more often earthward of geosynchronous orbit on the night side than on the dayside. Note that we do not have enough events to evaluate this asymmetry for stormtime cases.

\section{Conclusions}

We have inferred some features of the radial profiles of energetic particles through a statistical study of the geosynchronous particle flux responses to solar wind dynamic pressure enhancements, the majority of which (84\%) did not occur during the main or recovery phase of a magnetic storm. (Events that were sudden commencements reflect non-stormtime radial profiles). For protons and low-energy electrons, the primary response to a pressure enhancement is a flux increase. For relativistic electrons, the dominant response is a flux decrease, which implies that the radial profile of phase space density often peaks earthward of geosynchronous orbit.

While we are not able to conclusively infer the PSD radial profile for protons and low energy electrons, because of the decrease in $\mu$ values measured by fixed-energy particle detectors after a compression, there is a clear contrast with the inference that the peak of phase space density for relativistic electrons is within geosynchronous orbit. We have shown this statistically and without the use of a magnetic field model. Our result is in agreement with what was found by Chen et al. (2007), but we are not able to conclusively argue for an internal or external acceleration mechanism as the source of the PSD peak, as Chen et al. (2007) have done.

Although our work gives only indirect evidence of the source of relativistic electrons, and is limited by our simple equatorial-mirroring assumption, our results complement those of Green and Kivelson (2004) and Chen et al. (2005, 2007) by being from a different perspective and by not being magnetic field model dependent. Our results also confirm the results of Chen et al. (2007), in which the radial profiles of relativistic electrons during quiet times and storm times were found to peak earthward of geosynchronous orbit.

Acknowledgements. This work was supported by NASA grants NNG04GM98G and NNG05GF29G. We thank R. Lepping and R. Ogilvie for providing WIND solar wind data and D. McComas C. Smith for providing ACE solar wind data and J. Weygand for providing propagated solar wind data. We appreciate the LANL science team for providing the energetic particle flux data. We are grateful for the helpful discussion with Yuri Shprits at UCLA.

Topical Editor I. A. Daglis thanks two anonymous referees for their help in evaluating this paper.

\section{References}

Borodkova, N. L., Zastenker, G. N., Riazantseva, M., and Richardson, J. D.: Large and sharp solar wind dynamic pressure variations as a source of geomagnetic field disturbances at the geosynchronous orbit, Planet. Space Sci., 53, 25-32, 2005.

Chen, Y., Friedel, R. H. W., Reeves, G. D., Onsager, T. G., and Thomsen, M. F.: Multisatellite determination of the relativistic electron phase space density at geosynchronous orbit: Methodology and results during magnetically quiet time, J. Geophys. Res., 110, A10210, doi:10.1029/2004JA010895, 2005.

Chen, Y., Reeves, G. D., and Friedel, R. H. W.: The energization of relativistic electrons in the outer Van Allen radiation belt, Nature Phys., 3, 614-617, doi:10.1038/nphys655, 2007.

Friedel, R. H. W., Reeves, G. D., and Obara, T.: Relativistic electron dynamics in the inner magnetosphere-a review, J. Atmos. Solar Terr. Phys., 64, 265-282, 2002.

Green, J. C. and Kivelson, M. G.: Relativistic electron in the outer radiation belt: Differentiating between acceleration mechanisms, J. Geophys. Res., 109, A03213, doi:10.1029/2003JA010153, 2004.

Hilmer, R. V., Ginet, G. P., and Cayton, T. E.: Enhancement of equatorial energetic electron fluxes near $\mathrm{L}=4.2$ as a result of high speed solar wind stream, J. Geophys. Res., 105, 23311-23322, 2000.

Hudson, M. K., Elkington, S. R, Lyon, J. G., Marchenko, V. A., Roth, I., Temerin, M., Blake, J. B., Gussenhoven, M. S., and Wygant, J. R.: Simulation of radiation belt formation during storm sudden commencements, J. Geophys. Res., 102, 1408714102, 1997.

Hudson, M. K., Kress, B. T., Mueller, H., Zastrow, J. A., and Blake, J. B.: Relationship of the Van Allen radiation belts to solar wind drivers, J. Atmos. Solar Terr. Phys., 70, 708-729, 2008.

Iles, R. H. A., Meredith, N. P., Fasakerley, A. N., and Horne, R. B.: Phase space density analysis of the outer radiation belt energetic electron dynamics, J. Geophys. Res., 111, A03204, doi:10.1029/2005JA011206, 2006.

Iyemori, T. and Rao, D. R. K.: Decay of the Dst field of geomagnetic disturbance after substorm onset and its implication to storm-substorm relation, Ann. Geophys., 14, 608-618, 1996, http://www.ann-geophys.net/14/608/1996/.

Lee, D.-Y., Lyons, L. R., and Reeves, G. D.: Comparison of geosynchronous energetic particle flux responses to solar wind dynamic pressure enhancements and substorms, J. Geophys. Res., 110, A09213, doi:10.1029/2005JA011091, 2005.

Li, X., Baker, D. N., Temerin, M., Caytoy, T., Reeves, G. D., Araki, T., Singer, H., Larson, D., Lin, R. P., and Kanekal, S. G.: Energetic electron injections into the inner magnetosphere during Jan. 10-11, 1997 magnetic storm, Geophys. Res. Lett., 25, 25612564, 1998.

Li, X., Baker, D. N., Elkington, S., Temerin, M., Reeves, G. D., Belian, R. D., Blake, J. B., Singer, H. J., Peria, W., and Parks, G.: Energetic particle injections in the inner magnetosphere as a response to an interplanetary shock, J. Atmos. Solar Terr. Phys., 65, 233-244, 2003.

Li, X., Roth, I., Temerin, M., Wygant, J. R., Hudson, M. K., and Blake, J. B.: Simulation of the prompt energization and transport of radiation belt particles during the March 24, 1991 SSC, Geophys. Res. Lett., 20, 2423-2426, 1993.

Lyatsky, W. and Khazanov, G. V.: Effect of solar wind density 
on relativistic electrons at geosynchronous orbit, Geophys. Res. Lett., 35, L03109, doi:10.1029/2007GL032524, 2008.

Lyons, L. R.: Magnetospheric processes leading to precipitation, Space Sci. Rev., 80, 109-132, 1997.

Lyons, L. R. and Thorne, R.: Equilibrium structure of radiation belt electrons, J. Geophys. Res., 78(13), 2142-2149, 1973.

McAdams, K. L., Reeves, G. D., Friedel, R. H. W., and Cayton, T. E.: Multisatellite comparisons of the radiation belt response to the Geospace Environment Modeling (GEM) magnetic storms, J. Geophys. Res., 106, 10869-10882, 2001.

Onsager, T., Chan, A., Fei, Y., Elkington, S., Green, J., and Singer, H.: The radial gradient of relativistic electrons at geosynchronous orbit, J. Geophys. Res., 109, A05221, doi:10.1029/2003JA010368, 2004.

Selesnick, R. S. and Blake, J. B.: Dynamics of the outer radiation belt, Geophys. Res. Lett., 24, 1347-1350, 1997.

Selesnick, R. S. and Blake, J. B.: On the source location of radiation belt relativistic electrons, J. Geophys. Res., 105, 2607-2624, 2000.

Shi, Y., Zesta, E., Lyons, L. R., et al.: Effect of solar wind pressure enhancement on storm-time ring current asymmetry, J. Geophys. Res., 110, A10205, doi:10.1029/2005JA011019, 2005.

Shi, Y., Zesta, E., Lyons, L. R., et al.: Statistical study of solar wind dynamic pressure enhancement effect on dawn-todusk ring current asymmetry, J. Geophys. Res., 111, A10216, doi:10.1029/2005JA011532, 2006.

Summers, D., Ni, B. B., and Meredith, N. P.: Timescales for radiation belt electron acceleration and loss due to resonant waveparticle interactions: 2. Evaluation from VLF chorus, ELF hiss, and electromagnetic ion cyclotron waves, J. Geophys. Res., 111, A04207, doi:10.1029/2006JA011998, 2007.
Tsyganenko, N. and Stern, D. P.: Modeling the global magnetic field of the large scale Birkland current systems, J. Geophys. Res., 101, 27187-27198, 1996.

Taylor, M. G. G. T., Friedel, R. H. W., Reeves, G. D., Dunlop, M. W., Fritz, T. A., Daly, P. W., and Balogh, A.: Multisatellite measurements of electron phase space density gradients in the Earth's inner and outer magnetosphere, 109, A05220, doi:10.1029/2003JA010294, 2004.

Wang, C.-P., Lyons, L. R., Chen, M. W., and Wolf, R. A.: Modeling the quiet time inner plasma sheet protons, J. Geophys. Res., 106 , 6161-6178, 2001.

Wang, C., Liu, J. B., Huang, Z. H., and Richardson, J. D.: Response of the magnetic field in the geosynchronous orbit to solar wind dynamic pressure pulses, J. Geophys. Res., 112, A12210, doi:10.1029/2007JA012664, 2007.

Weimer, D. R., Ober, D. M., Maynard, N. C., Collier, M. R., McComas, D. J., Ness, N. F., Smith, C. W., and Watermann, J.: Predicting interplanetary magnetic field (IMF) propagation delay times using the minimum variance technique, J. Geophys. Res., 108, A11026, doi:10.1029/2002JA009405, 2003.

Wing, S. and Sibeck, D. G.: Effects of interplanetary magnetic field $\mathrm{z}$ component and the solar wind dynamic pressure on the geosynchronous magnetic field, J. Geophys. Res., 102, 72077216, 1997.

Wolf, R. A.: The quasi-static (slow-flow) region of the magnetosphere, in: Solar Terrestrial Physics, edited by: Carovillano, $\mathrm{R}$ L. and Forbes, J. M., pp. 303-368, D. Reidel, Norwell, Mass, 1983. 